\title{
Modal correction for fiber-coupling efficiency in free-space optical communication systems through atmospheric turbulence
}

\author{
Hanling $\mathrm{Wu}^{\mathrm{a}, *}$, Haixing Yan ${ }^{\mathrm{a}, 1}$, Xinyang $\mathrm{Li}^{\mathrm{b}, 2}$ \\ a Institute of Mechanics, Chinese Academy of Sciences, No. 15 Beisihuanxi Road, Beijing 100190, China \\ ${ }^{\mathrm{b}}$ Institute of Optoelectronics, Chinese Academy of Sciences, No. 350 mailbox, Shuangliu, Chengdu 610209, China
}

\section{A R T I C L E I N F O}

\section{Article history:}

Received 3 January 2009

Accepted 30 April 2009

\section{Keyword:}

Free-space optical communication

Atmospheric turbulence

Fiber coupling efficiency

Adaptive optics

Modal correction

\begin{abstract}
A B S T R A C T
High-speed free-space optical communication systems have recently used fiber-optical components. The coupling efficiency with which the received laser beam can be coupled into a single-mode fiber is noticeably limited by atmospheric turbulence due to the degradation of its spatial coherence. Fortunately, adaptive optics (AO) can alleviate this limitation by partially correcting the turbulencedistorted wavefront. The coupling efficiency improvement provided by Zernike modal AO correction is numerically evaluated. It is found that the first 3-20 corrected polynomials can considerably improve the fiber-coupling efficiency. The improvement brought by AO is compared with that brought by a coherent fiber array. Finally, a hybrid technique that integrates AO and a coherent fiber array is proposed. Results show that the hybrid technique outperforms each of the two above-mentioned techniques.
\end{abstract}

(c) 2009 Elsevier GmbH. All rights reserved.

\section{Introduction}

Free-space optical (FSO) communication through the turbulent atmosphere is currently being considered for various applications, e.g. as an alternative for fiber optic links between buildings, reconfigurable and mobile communication links for military operations, or ground-satellite optical communication [1,2]. Because of the high demands for data transmission rates of communication systems, future FSO communication systems must work with optical-fiber-based technology such as transmitter and receiver modules, erbium-doped fiber amplifiers (EDFAs), and multiplexer and demultiplexer units for wavelength division multiplexing, which allow higher link capacities and longer link distances. In these systems using single-mode fibers, the fibercoupling efficiency is one of the most significant issues. It represents the amount of energy that can be coupled into a single-mode fiber of a given diameter. However, atmospheric turbulence degrades the spatial coherence of a propagating beam and then limits the coupling efficiency [3].

Some techniques have been proposed to mitigate this degradation. One of them is adaptive optics (AO) [4], which measures the wavefront distortion induced by turbulence and rapidly compensates for it either by using deformable mirrors or by using a material with variable refractive property. Some investigations

\footnotetext{
* Corresponding author. Tel.:+8601082544248.

E-mail addresses: wuhanling@imech.ac.cn (H. Wu), hxyan@imech.ac.cn (H. Yan), xyli@ioe.ac.cn (X. Li).

1 Tel.: +8601082544248.

2 Tel.: +8602885101077
}

$[2,5,6]$ have been done to the improvement of coupling efficiency by using AO systems. Wilks et al. [5] introduced a wave-optics simulation code, which was used to model AO-based FSO communication systems. Their results showed that the coupling efficiency has an increase of $35 \%$ (from $20 \%$ to $55 \%$ ) by using an AO correction under moderate turbulence conditions. Based on the stochastic parallel gradient descent algorithm and 37 control channels, Plett et al. [6] used an AO system to compensate for static aberrations. They demonstrated a $12 \mathrm{~dB}$ improvement in the coupling efficiency in laboratory with this system. A similar system was also used to compare blind optimization with a conventional AO system based on wavefront conjugation, and it was found that using the coupling efficiency as a feedback signal in the AO system could provide better results [2].

Unlike those analyses of the above-mentioned literature, the fiber-coupling efficiency improvement with the so-called Zernike modal AO correction is theoretically investigated in this paper. Turbulence-distorted wavefronts are two-dimensional functions that can be decomposed into Zernike polynomials that are separable in angle and radius and form an orthogonal basis [7]. The effect of modal correction on distorted wavefronts is that some of the decomposition coefficients vanish. This type of correction is often preferable because a deformable mirror is able to reproduce a superposition of Zernike modes with a high accuracy [8]. For simplicity, here we restrict our attention to the case of a modal AO correction by totally removing some Zernike aberration modes. In this paper the temporal response of AO systems is not investigated and it is assumed that the control bandwidth is unlimited. We will show that an AO correction can increase the spatial coherence radius and then improve the coupling efficiency. 
A coherent fiber array (CFA) $[3,9]$ is a promising alternative to AO systems. It is formed by multiple apertures interfaced to separate single-mode fibers. The light phase in each fiber is adjusted before outputs of fibers are combined coherently. CFA-based transmitter and receiver structures can improve the fiber-coupling efficiency. These structures were evaluated by theory [3] and experiment [9], respectively. In this paper, improvements in the coupling efficiency provided by the two techniques, i.e. a modal AO correction and a coherent fiber array, are compared. Then, a hybrid technique is proposed. It incorporates the two above-mentioned techniques in an FSO system through atmospheric turbulence. It is shown that this hybrid technique can highly improve the coupling efficiency in comparison with using one technique alone.

\section{Zernike modal AO correction}

The Fried parameter and spatial coherence radius, the wavefront decomposition into Zernike polynomials and a generalization of the Fried parameter and spatial coherence radius are described in this section.

\subsection{Fried parameter and spatial coherence radius}

Uncorrected wavefronts can be characterized with use of the Fried parameter $r_{0}$, which corresponds to the diameter of the atmospheric coherence area. For constant $C_{n}^{2}$ and the plane wave [10],

$r_{0}=\left(0.423 C_{n}^{2} k^{2} L\right)^{-3 / 5}$,

where $k$ is wave number $2 \pi / \lambda$, $\lambda$ the light wavelength, $C_{n}^{2}$ the refractive index structure constant dependent on location, time and altitude and $L$ denotes thickness of a turbulent layer.

Spatial coherence radius $r_{c}$ is a specific distance transverse to the propagation direction where the average electric field correlation falls to $1 / e$ of its fully correlated value. For constant $C_{n}^{2}$ and plane wave, it is given by [10]

$r_{c}=\left(1.46 C_{n}^{2} k^{2} L\right)^{-3 / 5}$.

The Fried parameter $r_{0}$ is related to the plane wave spatial coherence radius $r_{c}$ by $r_{0}=2.1 r_{c}$.

\subsection{Wavefront description}

The turbulence-induced wavefront aberrations vary randomly in space and time. In order to characterize the spatial variation of a wavefront at a given time, it is useful to decompose the wavefront with respect to a set of basis functions. Zernike polynomials are an appropriate basis set. Using the definition given by Noll [7], a wavefront aberration can be written as

$\phi(r, \theta)=\sum_{i=1}^{\infty} a_{i} Z_{i}(r, \theta)$

where $a_{i}$ are coefficients of corresponding Zernike polynomials $\left(Z_{i}\right)$. The effect of modal correction on a wavefront is that some of the decomposition coefficients vanish. When the first $j$ Zernike terms have been corrected, the residual distortion in the corrected wavefront may be estimated with the Noll expression for the average variance over the wavefront surface [7]

$\Delta_{j}=\sum_{i=j+1}^{\infty}\left\langle\left|a_{i}\right|^{2}\right\rangle=\operatorname{coef}(j)\left(\frac{D}{r_{0}}\right)^{5 / 3}$, where $\langle\bullet\rangle$ denotes an ensemble average and $\operatorname{coef}(j)$ is the corresponding coefficient given by Noll for the correction degree $j$. For larger values of $j$, an approximate formula can be written as [7]

$\operatorname{coef}(j) \approx 0.2944 j^{-\sqrt{3} / 2}$.

\subsection{Generalized Fried parameter and spatial coherence radius}

In a similar way, it is possible to define a generalized Fried parameter $\rho_{0}$ corresponding to corrected wavefronts. The generalized Fried parameter after a modal AO correction is given by [11]

$\rho_{0}=0.286\left(\frac{3.44}{\operatorname{coef}(j)}\right)^{3 / 5} j^{-0.362} r_{0}$.

Similarly, the spatial coherence radius of a plane wave after a modal correction can be obtained as

$\rho_{c}=2.1 \rho_{0}$.

\section{Fiber-coupling efficiency for partially coherent light}

The fiber-coupling efficiency for partially coherent light is defined as the ratio of the average power coupled into the fiber $\left\langle P_{c}\right\rangle$ to the average power in the receiver aperture plane $\left\langle P_{a}\right\rangle$ and is given by $[3,12]$

$\eta=\frac{\left\langle P_{c}\right\rangle}{\left\langle P_{a}\right\rangle}=\frac{\left\langle\left|\int_{A} U_{i}(r) U_{m}^{*}(r) d r\right|^{2}\right\rangle}{\left\langle\int_{A}\left|U_{i}(r)\right|^{2} d r\right\rangle}$,

where $U_{i}(r)$ is the incident optical field in the receiver aperture plane and $U_{m}(r)$ the normalized fiber-mode profile. For plane wave and Kolmogorov power-law spectrum, a general expression for the fiber-coupling efficiency in a single-mode fiber is given by $[3,12]$

$$
\begin{aligned}
\eta= & \frac{8 a^{2}}{\pi} \int_{0}^{1} \int_{0}^{1} \exp \left[-a^{2}\left(x_{1}^{2}\right.\right. \\
& \left.\left.+x_{2}^{2}\right)\right] F\left(\frac{A_{R}}{A_{C}}\left(x_{1}^{2}+x_{2}^{2}\right), \frac{2 x_{1} x_{2}}{x_{1}^{2}+x_{2}^{2}}\right) x_{1} x_{2} d x_{1} d x_{2},
\end{aligned}
$$

where

$F(v, u)=\int_{0}^{\pi} \exp \left\{-v^{5 / 6}[1-u \cos (\theta)]^{5 / 6}\right\} d \theta$.

$A_{R}=\pi D_{R}^{2} / 4$ is the area of the receiver aperture, $D_{R}$ the receiver lens diameter, $A_{c}=\pi \rho^{2}$ the spatial coherence area of the incident plane wave and $\rho$ the spatial coherence radius given by $r_{c}$ or $\rho_{c}$. The ratio $A_{R} / A_{c}$ represents the number of speckles over the receiver aperture area. The parameter $a=\left(D_{R} / 2\right)\left(\pi W_{m} / \lambda f\right)$ is the ratio of the receiver lens radius to the radius of the backpropagated fiber mode. The optimum value of the couplinggeometry parameter $a$ maximizes the coupling efficiency and it depends on the number of speckles $A_{R} / A_{c}$. In the absence of turbulence, the incident plane wave is fully coherent and the optimum value of $a$ is 1.12 . It has been already shown that if the lens focal length is chosen to satisfy $a=1.12$ [3,12], corresponding to the optimum $a$ value for a fully coherent incident plane wave, the coupling efficiency obtained for other values of $A_{R} / A_{c}$ is not appreciably less than the maximum coupling efficiency that can be obtained by optimizing $a$. Thus, $a=1.12$ is used for the remainder of the following calculations.

An analysis of Eq. (10) shows that the coupling efficiency depends only on coupling-geometry parameter $a$ and the number of speckles over the receiver aperture area $A_{R} / A_{c}$. It is shown that 
the coupling efficiency decreases rapidly with increasing number of speckles over the receiver aperture at $a=1.12$. Thus, increasing the spatial coherence radius can reduce the number of speckles over the receiver aperture and then improve the coupling efficiency. AO can increase the spatial coherence radius by correcting the turbulence-distorted wavefront.

\section{Results and discussions}

\subsection{Coupling efficiency with/without modal correction}

From Eqs. (1), (2) and (9), the effects of atmospheric turbulence on the fiber-coupling efficiency can be investigated. Furthermore, with the help of Eq. (7), the improvement of the fiber-coupling efficiency by a modal correction can be also investigated. In order to quantify the relative improvement provided by a modal correction, an improvement factor of the coupling efficiency is defined as

Improvement factor $=\frac{\eta_{\text {corrected }, j}}{\eta_{\text {uncorrected }}}$,

where $\eta_{\text {corrected }_{j}}$ is the coupling efficiency after a correction of first $j$ polynomials while $\eta_{\text {uncorrected }}$ is the coupling efficiency without any degree of correction.

Fig. 1 shows the coupling efficiency and its improvement factor as a function of the turbulence strength for a link distance of $1000 \mathrm{~m}$ and various degrees of correction. Five levels of correction are chosen and they are 3,10,20, 50 and 80 . The receiver lens diameter was taken to be $10 \mathrm{~cm}$ and the wavelength is $1.55 \mu \mathrm{m}$. $j=0$ denotes there is no degree of phase correction. When there is no degree of correction, the coupling efficiency decreases rapidly with increasing $C_{n}^{2}$ because the number of speckles over the receiver aperture increases. With increase in the number of
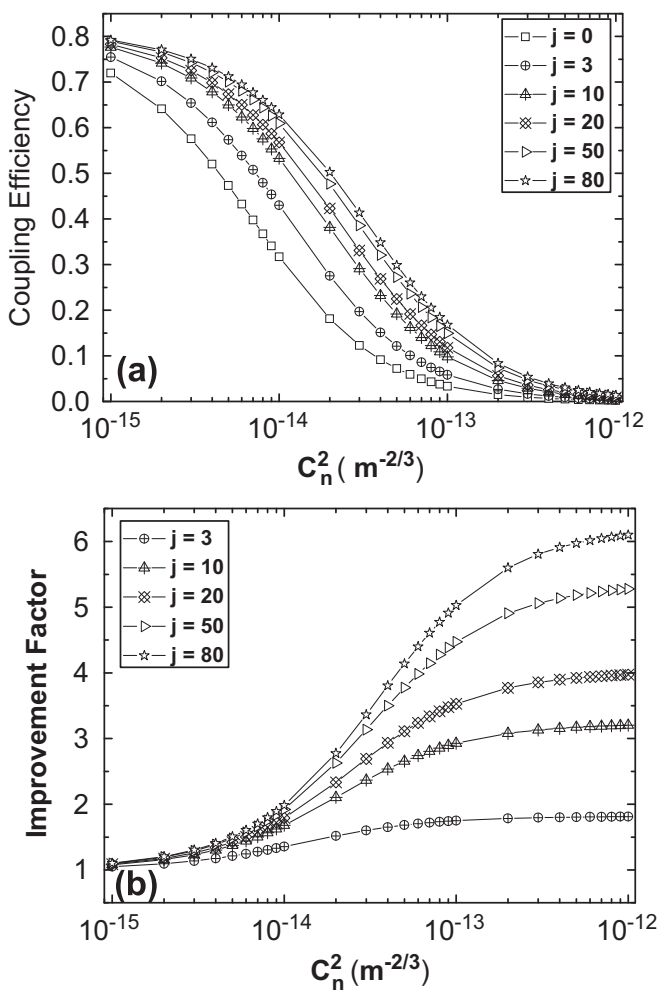

Fig. 1. Fiber-coupling efficiency and the efficiency improvement factor as a function of $C_{n}^{2}$ for different numbers of corrected polynomials. corrected polynomials, the increase in the fiber-coupling efficiency is significant under various turbulence conditions. In addition, all the different levels of correction can produce much better improvement of the coupling efficiency in a higher turbulence than in a weaker turbulence. The improvement factor increases with increase in turbulence strength. At $C_{n}^{2}=10^{-15} \mathrm{~m}^{-2 / 3}$, removing the first 80 Zernike polynomials improves the coupling efficiency by a factor 1.01 from 0.72 to 0.79 , while a factor 5.1 from 0.033 to 0.168 at $C_{n}^{2}=10^{-13} \mathrm{~m}^{-2 / 3}$. Furthermore, the improvement brought by 3-20 corrected polynomials is significantly better than others and the improvement with more than 20 corrected polynomials is minor. This shows that a low-order AO system can improve the coupling efficiency more effectively. Due to its low cost and simplicity, incorporation of a low-order AO system in an FSO communication system through turbulent atmosphere is relatively easy. Fig. 1(b) also shows that the improvement factor significantly increases with increasing $C_{n}^{2}$ in a moderate turbulence $C_{n}^{2}=10^{-14}-10^{-13} \mathrm{~m}^{-2 / 3}$.

Fig. 2 shows the coupling efficiency as a function of link distance for three different $C_{n}^{2}$ values and the first 50 Zernike polynomials corrected. These $C_{n}^{2}$ values represent weak, moderate and strong turbulence conditions, respectively. A link distance of zero gives the maximum coupling efficiency of 0.81 that can be obtained with a fully coherent plane wave. Under a strong turbulence condition, a $1000 \mathrm{~m}$ link has a coupling efficiency of less than 0.03 , indicating the need to allocate a significant amount of the link power budget to fiber-coupling efficiency. After the first 50 Zernike polynomials are compensated, the coupling efficiency reaches 0.15 , which corresponds to a $2500 \mathrm{~m}$ link under moderate turbulence conditions. Thus, increasing the transmission power is no longer needed and the link distance can be increased.
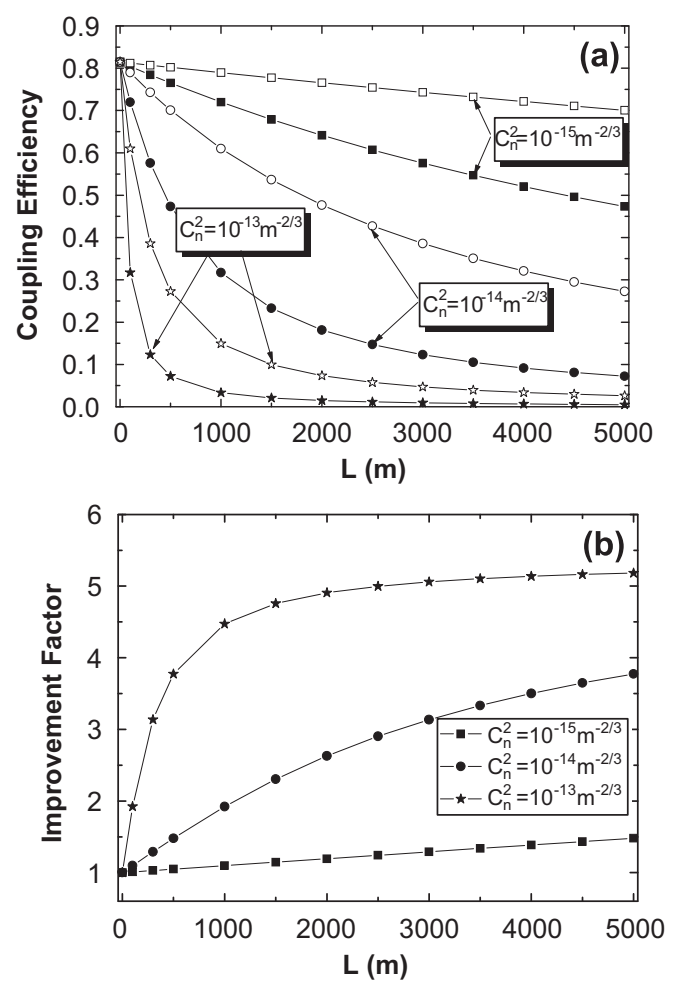

Fig. 2. Fiber-coupling efficiency and the efficiency improvement factor as a function of link distance for three different values of $C_{n}^{2}$ and $j=50$. In Fig. 2(a), solid symbols denote the results without any degree of compensation while hollow symbols denote the results with the first 50 Zernike polynomials corrected. 


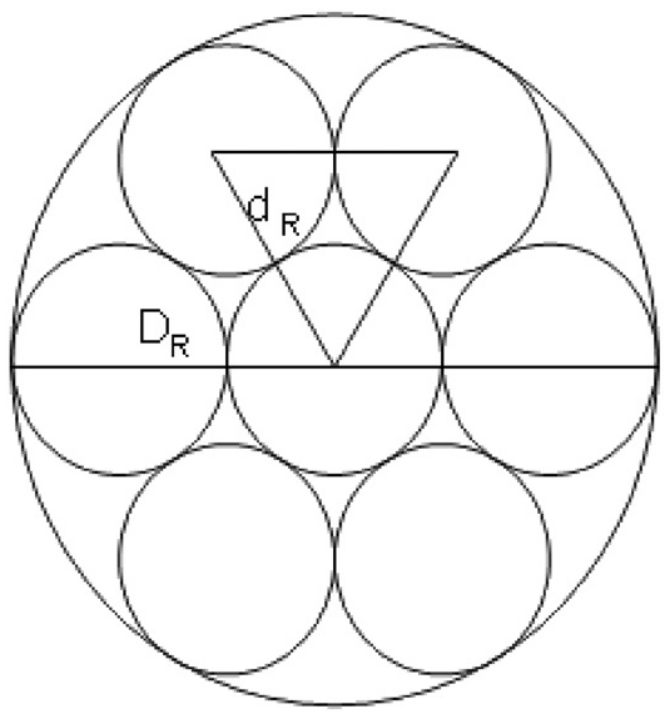

Fig. 3. Hexagonal close-packed arrangement of a coherent fiber array. The seven small circles represent subapertures of diameter $d_{R}$ and the large circle represents a single receiver aperture of diameter $D_{R}$.

\subsection{Comparison of the fiber-coupling efficiency improvement} brought by modal correction with that brought by a coherent fiber array

In order to compare the fiber-coupling efficiency improvement brought by a modal correction with that brought by a coherent fiber array (CFA), a coherent fiber array with seven subapertures, which are arranged in a hexagonal close-packed array as in the literature [3] is considered. This fiber array is shown in Fig. 3. Each subaperture contains a lens that couples the received light into a single-mode fiber and its diameter is $d_{R}=D_{R} / 3$.

The results for a comparison are shown in Fig. 4. Fig. 4(a) shows the coupling efficiency as a function of $C_{n}^{2}$ for a link distance of $1000 \mathrm{~m}$. Four corrected levels are chosen and they are 20, 50,120 and 300 corrected polynomials. At $C_{n}^{2}=10^{-14} \mathrm{~m}^{-2 / 3}$, the improvement factor provided by 20 corrected polynomials is larger than that by a coherent fiber array with seven subapertures. The coherent fiber array improves the coupling efficiency from 0.32 to 0.52 , while removing the first 20 Zernike polynomials improves the coupling efficiency from 0.32 to 0.57 . But, as $C_{n}^{2}$ increases, the improvement provided by 20 corrected polynomials becomes gradually smaller than that by a coherent fiber array with seven subapertures. In contrast, the improvement by 120 corrected polynomials is comparable to that of a coherent fiber array at a larger value of $C_{n}^{2}$. Fig. $4(\mathrm{~b})$ shows the coupling efficiency as a function of link distance for $C_{n}^{2}=10^{-13} \mathrm{~m}^{-2 / 3}$. The comparison is similar to Fig. 4(a).

From Eq. (10), it is shown that the coupling efficiency is improved only by reducing the number of speckles $A_{R} / A_{c}$ over the receiver aperture at a given $a=1.12$. In order to reduce the number of speckles $A_{R} / A_{c}$, AO increases the spatial coherence radius by correcting the turbulence-distorted wavefront. In contrast, a coherent fiber array reduces the number of speckles over each subaperture by using a coherent fiber array with a smaller receiver aperture. Certainly, the improvements are related with the number of corrected polynomials and the structure of coherent fiber array.

\subsection{Improvement of coupling efficiency by incorporating $A O$ and CFA} in FSO through atmospheric turbulence

As mentioned above, both $\mathrm{AO}$ and CFA can improve the coupling efficiency by reducing the number of speckles over the
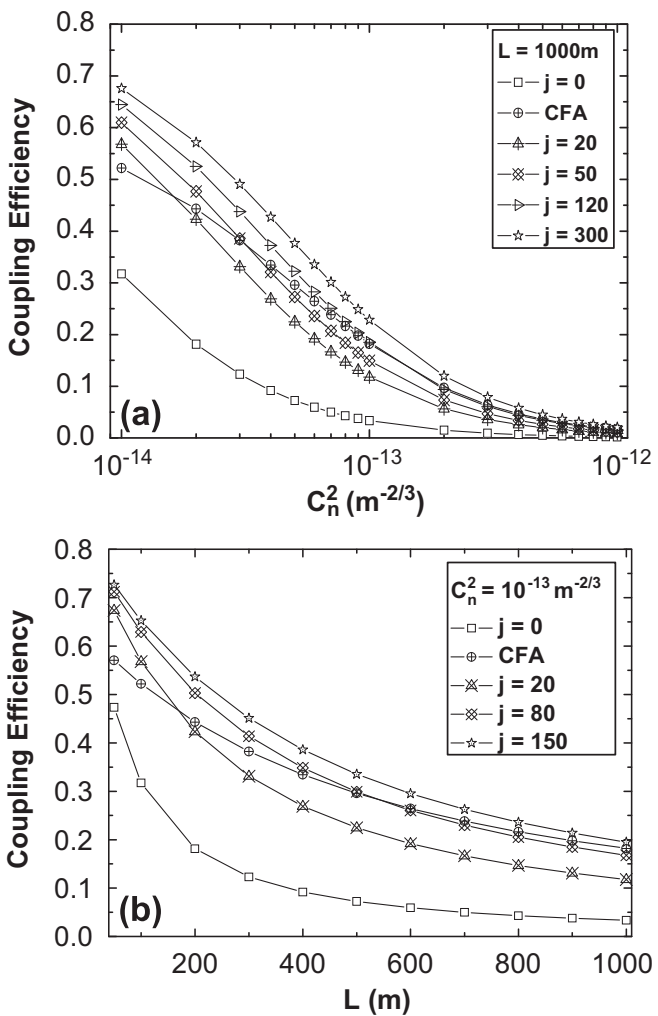

Fig. 4. Fiber-coupling efficiency as a function of $C_{n}^{2}$ (a) and link distance (b), where $D_{R}=10 \mathrm{~cm}, a=1.12$ and $\lambda=1.55 \mu \mathrm{m}$. CFA denotes the coherent fiber array result.
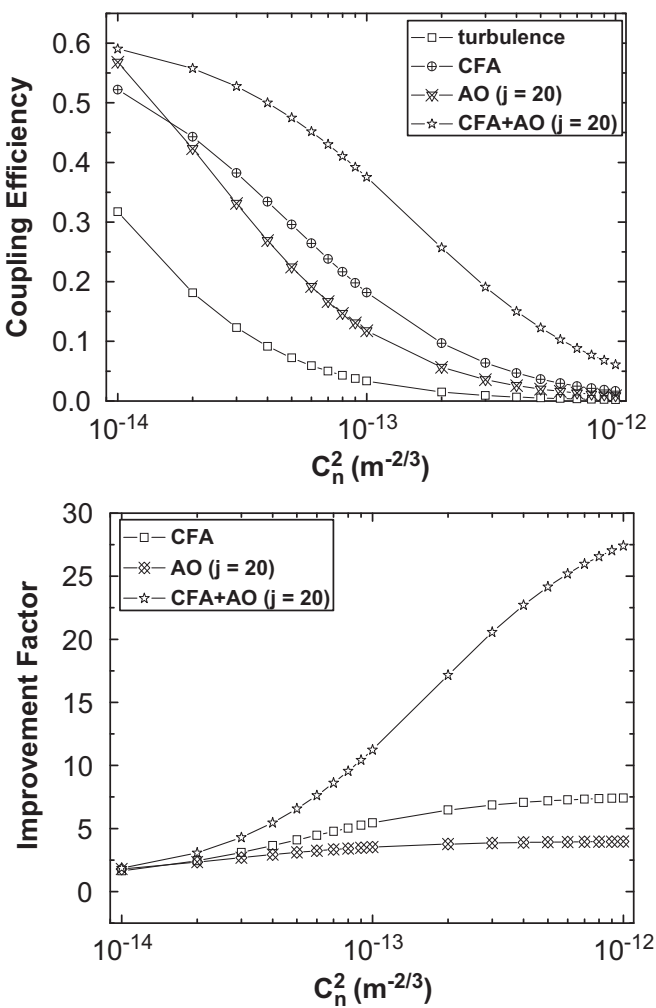

Fig. 5. Fiber-coupling efficiency and the improvement factor as a function of $C_{n}^{2}$ for a link distance of $1000 \mathrm{~m}, D_{R}=10 \mathrm{~cm}, a=1.12$ and $\lambda=1.55 \mu \mathrm{m}$. AO $(j=20)$ denotes a modal correction with 20 Zernike polynomials removed. 
receiver aperture. In this section, the coupling efficiency of an optical-fiber-based FSO communication system incorporating both the above-mentioned techniques is investigated. A hybrid technique is proposed to increase the coupling efficiency of an FSO system. It integrates $\mathrm{AO}$ and CFA as a receiver structure and the structure of the CFA in it is the same as in Section 4.2.

The coupling efficiency improvement provided by the hybrid technique is shown in Fig. 5. Fig. 5 shows the coupling efficiency is significantly larger than that provided by AO or CFA alone. At $C_{n}^{2}=10^{-13} \mathrm{~m}^{-2 / 3}$, the improvement factors provided by an $\mathrm{AO}$ with 20 corrected polynomials, a CFA and incorporating both techniques are 4, 6 and 11, respectively. The coupling efficiency increases from 0.03 to 0.38 by utilizing the hybrid technique. The hybrid technique can be utilized to reduce the power requirement to the transmitter and the responsibility requirements to the receiver while maintaining the same link distance.

Although incorporating AO and CFA can significantly improve the coupling efficiency for a free space communication system through atmospheric turbulence, it results in a fairly complicated and expensive receiver structure. Hence, its applications are limited and a trade-off between the benefit of this hybrid technique and its cost should be established.

\section{Summary}

The fiber-coupling efficiency for light propagated through the atmospheric turbulence with/without a Zernike modal AO correction has been numerically evaluated. Results show that an AO system can significantly increase the coupling efficiency because of the increased spatial coherence of the incident light. On increasing the number of corrected polynomials, the improvement also increases. But, the improvement with the first 20 corrected polynomials is major and this shows that a low-order AO system can increase the coupling efficiency substantially.

The coupling efficiency improvement provided by a Zernike modal AO correction is also compared with that provided by a coherent fiber array. Under relatively weaker turbulence condi- tions, the improvement provided by a modal correction is better than that provided by a coherent fiber array. But under relatively stronger turbulence conditions, the improvement provided by more than 120 corrected polynomials can be comparable to that provided by a CFA. Both AO and CFA increase the coupling efficiency by reducing the number of speckles over the receiver aperture. A hybrid technique is proposed to increase the coupling efficiency. It integrates AO and CFA as a receiver structure. The improvement brought by the hybrid technique is superior to that brought by each of the two above-mentioned techniques. Under some conditions, it can improve the coupling efficiency by a factor of 11 , from 0.03 to 0.38 . But this hybrid technique and its system may be fairly complicated and expensive.

\section{References}

[1] L.Y. Wan, L.R. Liu, J.F. Sun, On-ground simulation of optical links for free-space laser communications, Optik, in press, doi:10.1016/j.jjleo.2008.07.002.

[2] T. Weyrauch, M.A. Vorontsov, J.W. Gowens, T.G. Bifano, Fiber coupling with adaptive optics for free-space optical communication, Proc. SPIE 4489 (2002) 177-184.

[3] Y. Dikmelik, F.M. Davidson, Fiber-coupling efficiency for free-space optical communication through atmospheric turbulence, Appl. Opt. 44 (2005) 4946-4952.

[4] R.K. Tyson, Principles of Adaptive Optics, Academic Press, New York, 1991.

[5] S.C. Wilks, J.R. Morris, J.M. Brase, S.S. Olivier, J.R. Henderson, C. Thompson, M. Kartz, A.J. Ruggerio, Modeling of adaptive optics-based free-space communication systems, Proc. SPIE 4421 (2002) 121-128.

[6] M.L. Plett, P.R. Barbier, D.W. Rush, Compact adaptive optical system based on blind optimization and a micromachined membrane deformable mirror, Appl. Opt. 40 (2001) 327-330.

[7] R.J. Noll, Zernike polynomials and atmospheric turbulence, J. Opt. Soc. Am. 66 (1976) 207-211.

[8] R.K. Tyson, Using the deformable mirror as a spatial filter: applications to circular beams, Appl. Opt. 21 (1982) 787-793.

[9] H. Bruesselbach, M.L. Minden, S. Wang, D.C. Jones, M.S. Mangir, A coherent fiber array based laser link for atmospheric aberration mitigation and power scaling, Proc. SPIE 5338 (2004) 90-101.

[10] L.C. Andrews, R.L. Phillips, Laser Beam Propagation through Random Media, SPIE Press, Bellingham, 2005.

[11] M.P. Cagigal, V.F. Canales, Generalized Fried parameter after adaptive optics partial wave-front compensation, J. Opt. Soc. Am. A 17 (2000) 903-910.

[12] P.J. Winzer, W.R. Leeb, Fiber coupling efficiency for random light and its applications to lidar, Opt. Lett. 23 (1998) 986-988. 